\title{
Building a Stronger CASA: Extending the Computers Are Social Actors Paradigm
}

\author{
Andrew Gambino ${ }^{1}$ (D) Jesse Fox $^{2}$ (D), and Rabindra (Robby) A. Ratan ${ }^{3}$ (D) \\ 1 The Donald P. Bellisario College of Communications, Pennsylvania State University, State College, \\ Pennsylania, United States of America \\ 2 School of Communication, Ohio State University, Columbus, Ohio, United States of America \\ 3 Department of Media and Information, Michigan State University, East Lansing, Michigan, United States \\ of America
}

\section{Abstract}

The computers are social actors framework (CASA), derived from the media equation, explains how people communicate with media and machines demonstrating social potential. Many studies have challenged CASA, yet it has not been revised. We argue that CASA needs to be expanded because people have changed, technologies have changed, and the way people interact with technologies has changed. We discuss the implications of these changes and propose an extension of CASA. Whereas CASA suggests humans mindlessly apply human-human social scripts to interactions with media agents, we argue that humans may develop and apply human-media social scripts to these interactions. Our extension explains previous dissonant findings and expands scholarship regarding human-machine communication, human-computer interaction, human-robot interaction, human-agent interaction, artificial intelligence, and computer-mediated communication.

Keywords: human-machine communication, human-computer interaction, human-robot interaction, media equation, computers are social actors (CASA)

\section{Introduction}

Theoretical development that explains and predicts human responses to social technologies has reached somewhat of a stalemate. The computers are social actors framework (CASA; Nass \& Moon, 2000; Nass et al., 1994), derived from the media equation (Reeves \& Nass, 1996), suggests that humans treat media and computers like real people, mindlessly applying scripts for interacting with humans to interactions with social technologies. 
CASA is often employed to guide research situated in human-machine communication (HMC), human-computer interaction (HCI), human-robot interaction (HRI), humanagent interaction (HAI), and media effects. These subfields, and the CASA research within, have grown over the past three decades due to technological advances and wider adoption of social technologies in areas such as health care, education, and the domestic sphere (e.g., Baylor, 2011; Fortunati, 2018; Kenny et al., 2008; Takayama, 2015). As computers, machines, and media have become more complex, more variable in form, and more integrated into our lives, the theoretical limitations of CASA have become more apparent.

Because of these changes, we propose an extension of CASA that accounts for ongoing changes in people, in technologies, and in the nature of their interactions. We argue that humans have developed more specified scripts for interacting with media. As a result, when humans are mindlessly interacting with media, they do not necessarily implement social scripts associated with human-human interactions as predicted by CASA. Instead, given a deeper and broader realm of experience, humans may implement scripts they have developed for interactions specific to media entities.

To support our argument, we will investigate the existing CASA literature. First, we will explain how technologies can serve as social actors and what characteristics cue human users to their social potential. Next, we will introduce CASA and review research within the paradigm, with a particular focus on studies with theoretical implications for HMC. Next, we discuss how changes in people, changes in technologies, and changes in human relationships with technologies indicate a need to revisit CASA, particularly in light of findings that challenge CASA's claims. Based on the preceding evidence, we argue for our theoretical extension to CASA: that humans may mindlessly apply human-media scripts just as they mindlessly apply human-human scripts to social interactions with technologies. In closing, we draw attention to the implications of our proposed extension of CASA, and how it enables theory building in human-machine communication.

\section{When Machines Act Human: Media Agents as Social Interactants}

As technologies have become more interactive and replaced tasks previously performed by humans, designers have attempted to minimize the cognitive effort it takes to use them. One method is to capitalize on users' existing mental models and mimic natural forms of social interaction, creating interfaces that reflect patterns of human communication to enhance usability (Nass \& Brave, 2005; Shneiderman et al., 2017). Because CASA is rooted in humans' understanding of social interaction, it is well-suited to inform research on the design and implementation of social technologies across HMC, HCI, HRI, HAI, and related fields.

To engage with CASA, it is necessary to clarify the scope of the framework and its boundary conditions. Importantly, CASA does not apply to every machine nor every social technology; Nass and colleagues have described two essential criteria for a technology that serve as boundary conditions for CASA's application. The first is social cues. Nass and Moon (2000) stated that "individuals must be presented with an object that has enough cues to lead the person to categorize it as worthy of social responses" (p. 83). Although this implies a boundary condition of "enough" social cues, unfortunately it is not a clearly defined one. 
Given that perceptions of social potential vary from person to person and situation to situation (Waytz et al., 2010), we cannot establish objective, universal parameters of what constitutes "enough" cues. For example, shapes resembling eyes and a mouth can be sufficient to trigger a social response from a baby, but given adults' more sophisticated brains, they may not perceive the same set of shapes as indicating social potential.

The second requisite characteristic is sourcing. Nass and Steuer (1993) clarified that CASA tests "whether individuals can be induced to make attributions toward computers as if the computers were autonomous sources" (p. 511). Even in naming their framework, Nass and colleagues make a meaningful choice in declaring that "computers are social actors." This distinction is important because computers and technologies in general often serve as channels or conduits for human-human communication. The ability to enact and be perceived as a source of communication, rather than merely transmit it, indicates that a technological artifact has a degree of agency and is more than merely a channel (e.g., Sundar \& Nass, 2000). Thus, for the sake of clarity and specificity, we conceptualize the types of technologies relevant to CASA as media agents. We define a media agent as any technological artifact that demonstrates sufficient social cues to indicate the potential to be a source of social interaction.

Thus, we employ the conceptualization of media agents to distinguish these entities from machines and technologies that are beyond the scope of CASA (see Guzman, 2018; Lewis et al., 2019, footnote 2). For example, adding a humanlike cue to a simple machine (e.g., gluing googly eyes to a stapler) does not indicate sufficient potential to be a source in social interaction. Media agents encompass a wide variety of technologies such as conversational agents, including voice assistants (e.g., Siri, Alexa), embodied conversational agents, and chatbots; virtual agents (e.g., computer-controlled video game characters); smart devices with social interfaces (e.g., a smart refrigerator), including wearables (e.g., Apple watch); and social robots (e.g., Paro, Aibo). We anticipate the number and complexity of media agents to grow as advancements are made in the technologies that power them, such as natural language processing and neural networks.

\section{The Computers Are Social Actors Framework}

The CASA framework was derived from Reeves and Nass's (1996) media equation; together, these have been referred to as the social responses to communication technologies (SRCT) approach (e.g., Sundar \& Nass, 2000). In their original book The Media Equation: How People Treat Computers, Television, and New Media Like Real People and Places, Reeves and Nass (1996) argue that humans' "old brains" do not have an evolved mechanism to automatically distinguish mediated representations from their real-life counterparts. When mediated representations mimic real life, humans respond to them naturally and mindlessly (Reeves \& Nass, 1996). These mindless responses extend to social interactions: When media depict social characteristics, humans treat them in a social manner rather than exerting the cognitive effort to determine how to respond (Reeves \& Nass, 1996). Thus, people will assign computers personality traits, apply stereotypes and norms, and make judgments and inferences as if the computers were human, even though they understand that computers are not human (Reeves \& Nass, 1996). 
CASA emerged from the media equation, focusing more specifically on interactions with digital technologies. CASA focuses more narrowly on conditions wherein technologies could be perceived similarly to humans: as social actors capable of agentic communication with a human user (Nass \& Moon, 2000; Nass \& Steuer, 1993; Nass et al., 1994).

\section{Social Scripts}

CASA argues that people respond mindlessly to media agents and thus communicate with them similarly to how they would communicate with another human. This implies that human users have a pre-existing mental model of how they would communicate with another human in a similar situation (Nass \& Moon, 2000). Mental models for interacting with others are referred to as social scripts (Honeycutt \& Bryan, 2011; Schank \& Abelson, 1977).

Over time, people encounter highly similar social situations; based on these experiences, they develop knowledge structures representing common series of sequenced behaviors. These social scripts are retained in memory and activated when relevant situations arise (Schank \& Abelson, 1977). Scripts provide useful heuristics that help humans navigate rote interactions, such as ordering a beer at a local bar. Existing scripts can also be extrapolated and applied to novel yet related situations, such as ordering a Jawa Juice at the Mos Eisley Cantina on Tatooine.

The heuristic activation of social scripts underlies the CASA framework. As Nass and Moon (2000) argued:

We can conclude that individuals are responding mindlessly to computers to the extent that they apply social scripts-scripts for human-human interactionthat are inappropriate for human-computer interaction, essentially ignoring the cues that reveal the essential asocial nature of the computer. (p. 83)

In other words, rather than questioning why a computer is demonstrating social behavior, humans follow the relevant human social script that has been mindlessly activated. CASA research has investigated how a variety of social scripts for human-human interaction are applied to human interactions with media agents.

\section{Findings Supporting CASA}

Several studies have demonstrated support for the CASA framework, including dozens of studies conducted by Nass and colleagues in the late 1990s. In these studies, the media agents were typically desktop computers that interacted using preprogrammed text-based or voice-based responses. For example, when participants were placed on the same team as a computer for a task, they rated the computer more favorably than if the computer was not labeled a teammate (Nass et al., 1996). In another study, participants encountering machines with male or female voices applied sex stereotypes to the machines, rating the female-voiced machine more expert at love and relationships and the male-voiced machine more knowledgeable about computers (Nass et al., 1997). Participants prefer computers 
that flatter them more than those that do not (Fogg \& Nass, 1997) and evaluate a computer more positively if the computer requests the evaluation rather than providing an evaluation separately on paper (Nass et al., 1994).

As computers and media agents became more complex than text on a screen, CASA retained its predictive validity in a wide range of contexts, including anthropomorphic interfaces (E. J. Lee, 2010b), embodied agents (Hoffmann et al., 2009; K. M. Lee et al., 2006), mobile phones (Carolus et al., 2018), and voice-based navigational systems (Nass et al., 2005). For example, K. M. Lee et al. (2006) found that, similar to interpersonal interactions, a key feature of positive interactions with embodied agents is their ability to communicate through touch. Nass et al. (2005) found that matching a driver's emotion to a car's voicebased emotion led to better performance in a driving simulator. The growing variety of media agents, however, led to research that supported different explanatory mechanisms for the effects of CASA (e.g., Gong \& Nass, 2007).

In recent years, the CASA framework has continued to garner support in studies examining more advanced technologies. Ho et al. (2018) found that the positive effects of interpersonal emotional disclosure applied to interactions with perceived chatbots. In HRI, studies adopting the CASA framework have demonstrated the effectiveness of human persuasive strategies (S. A. Lee \& Liang, 2016, 2019) and politeness (Srinivasan \& Takayama, 2016). Additionally, autonomous vehicles are perceived more positively if their voice agent's sex was stereotypically matched with its style of communication (i.e., informative male or sociable female; S. Lee et al., 2019). Results from CASA-framed studies continue to inform our understanding of communication phenomena (e.g., Hoffmann et al., 2009; Von der Pütten et al., 2010).

Although these studies have found support for CASA, several studies challenge its claims (e.g., Johnson \& Gardner, 2007; M. K. Lee et al., 2012; Pfeifer \& Bickmore, 2011) or suggest moderating factors (e.g., Chiasson \& Gutwin, 2005; E. J. Lee, 2009; E. J. Lee, 2010a; E. J. Lee, 2010b). We consider two overarching explanations for why CASA's claims are not supported. First, these studies did not establish whether participants were interacting mindlessly. It is possible that participants conducted a sort of Turing test, determined that this social agent was not a human, but a machine, and then deliberately treated it as such. A second possibility that we will explore in more depth here is that humans have developed and mindlessly apply distinct scripts for interacting with media agents that diverge from scripts for human-human interaction.

\section{Three Decades of Changes in People, Media Agents, and Their Interactions}

In the three decades since CASA was introduced, significant changes have taken place at both the societal and technological level. Because the focus of CASA is on how humans interact with emergent technologies, changes in both humans and technologies must be accounted for by predictive theory. We argue that such changes represent a shift in the sociocultural context in which CASA is applied, that these changes are ongoing, and that they drive the need to extend the CASA paradigm. 


\section{People Have Changed: Knowledge and Experiences With Media Agents}

Computational technologies have become increasingly integrated into the daily lives of people across the globe in the past 30 years. Particularly in economically advanced, industrialized nations, computers have gone from one-per-household to one-per-pocket. For example, household computer ownership in the United States increased from less than $40 \%$ in 1997 to over 80\% in 2016 (Ryan \& Lewis, 2017). Adult smartphone ownership in the United States grew from 35\% in 2011 to $81 \%$ in 2019, with overall cell phone use at 96\% in 2019 (Pew Research Center, 2019). These changes coincide with sharp increases in educational attainment (Ryan \& Bauman, 2016), information technology jobs (Beckhusen, 2016), and regular computer use across job sectors (Hipple \& Kosanovich, 2003). Based on these changes, we conclude that people's knowledge of and experiences with computers, and media agents by extension, have increased dramatically. Given growing exposure to and familiarity with media agents in recent years, people are likely to have changed in ways that complicate CASA.

Indeed, some studies within the CASA paradigm have supported the potential importance of individual differences such as education or experience with technology. From its earliest iterations, Nass and colleagues argued that some individual differences could be important when testing CASA, including demographics (e.g., level of education) and knowledge of technology (Nass \& Steuer, 1993). Related findings suggest that CASA effects are moderated by factors such as previous computer experience (Johnson \& Gardner, 2007) and that a person's expectations of media agents (e.g., social robots) vary based on their experience (Horstmann \& Krämer, 2019). Experience is relevant to CASA's assumption of mindlessness given that experience might determine whether a mindless or mindful response is triggered when encountering a media agent. Relatedly, characteristics of the media agent are also likely to affect the human response.

\section{Technologies Have Changed: Anthropomorphism}

Technological advances including expanded modalities for interaction, refined graphics, and faster computing power have increased the capacity for more human-like features in media agents. Many media agents have thus become more anthropomorphic in the way they behave or how they appear. Anthropomorphism is the perception of human traits or qualities in an entity and indicate its potential for social interaction (Breazeal, 2003; Waytz et al., 2010). People may perceive human-like appearance, sounds, or other sensory cues in a media agent (i.e., form anthropomorphism) or human-like actions (i.e., behavioral anthropomorphism; Nowak \& Fox, 2018).

Anthropomorphism is a key determinant of how media agents are evaluated (Blascovich et al., 2002; Gong \& Nass, 2007; E. J. Lee, 2010a, 2010b; Rosenthal-Von der Pütten \& Krämer, 2014). The study of anthropomorphism within the CASA paradigm has suggested generally positive effects (de Graaf \& Allouch, 2013). For example, Gong (2008) showed anthropomorphism has a positive, linear effect on perceptions of a digital representation's competence and trustworthiness. Moreover, Gong and Nass found that participants took more time processing information before making judgments of an agent with mismatched 
anthropomorphic features (i.e., face and voice) than an agent exhibiting matching anthropomorphic cues. This finding suggests that higher levels of anthropomorphism facilitate faster processing and may be more likely to evoke predicted CASA effects.

\section{Interactions Between Humans and Media Agents Have Changed}

Aside from their independent factors, there are two key ways that interactions between humans and media agents have changed over time. The first is tied to affordances. Affordances are the inherent functional attributes of an object that indicate possible actions by a user (Gibson, 1979). More specifically, social affordances indicate that an object has the capacity to accommodate communication (Fox \& McEwan, 2017). In terms of CASA, social affordances are relevant to understanding how humans will interpret the social potential of a media agent and if they will perceive it as a source rather than a channel.

In the past three decades, the social affordances of media agents have advanced. For example, greater memory capacity and more sophisticated artificial intelligence have facilitated increasing personalization, meaning that media agents can offer more tailored feedback to the user (Fox \& McEwan, 2017). Relevant to CASA, one study examined ongoing interactions with a social robot and found that participants interacting with a robot exhibiting higher personalization led to greater social responses (M. K. Lee et al., 2012). Additionally, children learned more over a 2-week period from a robot that personalized its behaviors than a non-personalized robot (Baxter et al., 2017).

Further, bandwidth, or the array of communicative cues that are enabled, has expanded greatly from the text-based interactions of the past (Fox \& McEwan, 2017). For example, modern robots can convey facial expressions, gestures, haptics, and proxemic cues. Given the broadened capacities of social affordances, media agents are providing more social cues, likely activating a broader range of social scripts among human users and demonstrating greater social potential to users. Moreover, studies have found that media agents are received more positively when they display cues that are more social (Pfeifer \& Bickmore, 2011) or socially appropriate (Gratch et al., 2007).

The second key factor is time. Given the increased accessibility and adoption of computers and smartphones by organizations and individuals (Hipple \& Kosanovich, 2003; Pew Research Center, 2019; Ryan \& Lewis, 2017), people interact with media agents far more frequently than they did three decades ago. Additionally, it has become more common for people to have repeated, ongoing interactions with media agents, such as Amazon's Alexa, to fulfill utilitarian as well as social needs (Ammari et al., 2019; McLean \& Osei-Frimpong, 2019). Improvements in social robot design also aim to facilitate long-term interactions (Leite et al., 2013) and acceptance into everyday settings (de Graaf et al., 2019).

Ongoing and long-term interactions present the opportunity for individuals to develop relationships with media agents similar to those with humans (Bickmore \& Cassell, 2001; Bickmore \& Picard, 2005). Longitudinal studies suggest that the relationship between a human and a media agent may change through ongoing interactions (see Leite et al., 2013, for a review). For example, Kim and Lim (2019) found that participant trust and partnership with a collaborative smartphone agent did not exist initially but were developed over 2 months of interactions. Moreover, Serholt and Barendregt (2016) found that children's social responses to robots decreased over time, suggesting that human-human interaction 
scripts began to fade or were applied less frequently as robots did not meet children's expectations for social interaction. Collectively, changes in the social affordances of media agents and the relationships they encourage indicate that CASA may lack explanatory power for modern users, media agents, and human-media agent interactions and relationships.

\section{Developing Scripts for Human-Media Agent Interaction}

These changes provide historical rationale to readdress CASA. Additionally, considerable evidence suggests that humans perceive media agents differently from how they perceive humans (e.g., Blascovich et al., 2002; Fox et al., 2015; Krämer et al., 2012). For example, people have distinct initial expectations for interactions with media agents (Edwards, 2018; Edwards et al., 2019; Spence et al., 2014). Different expectations and responses to humans and media agents can serve as evidence to simply refute CASA; that is, we may not treat media agents like people. Alternatively, we consider that through more social, frequent, and ongoing interactions with media agents, people may develop and apply specific scripts for interactions with media agents.

Extending CASA to incorporate scripts derived from human-media agent interaction addresses counterintuitive findings, accounts for the sociotechnological changes of the last three decades, and broadens CASA's theoretical scope. Arguably, humans need different mental models and scripts specific to media agents, or social phenomena related to the media agent, to best handle unmet expectations and maximize their own efficiency navigating novel conditions of interactions with media agents (e.g., media agents lack feelings). Results of longitudinal studies provide additional evidence that humans develop and apply scripts for interactions with media agents. Responses to social cues change upon multiple interactions with media agents, which suggests the development of scripts, and the resultant responses are systematic (Baxter et al., 2017; Bickmore \& Picard, 2005; Kim \& Lim, 2019; Krämer et al., 2011; M. K. Lee et al., 2012; Pfeifer \& Bickmore, 2011). The systematic responses to social cues, post-change, suggest that media-derived scripts, just like human-human scripts, are applied mindlessly in interactions with media agents. Over time, we have learned to acknowledge media agents and their affordances in interactions, and we have developed more nuanced scripts for interactions with media agents. Thus, we suggest extending CASA to include scripts derived from interactions with media agents.

When CASA's assertions were being formulated, human-media agent interactions were rare and lean compared to the current landscape in which media are pervasive and rich. At that time, the presentation and experience of social affordances were more limited because of the technology powering media agents. Advances in technologies such as natural language processing, neural-networks, and raw computing power allow for social affordances of modern media agents to manifest in a wider variety of forms and aptitudes. For example, unique data collection and processing power allows media agents to personalize at a qualitatively different level than humans. Hence, the study of social responses should not be restricted by a focus on human correlates or similarities. In this way, researchers can also avoid reifying face-to-face communication as the gold standard for HMC and being constrained by the limitations of human interactions (e.g., Fortunati, 2005; Spence, 2019). Instead, researchers can explore why communication with a media agent may be preferred 
over communication with a human. Removing this anthropocentric bias from CASA should allow researchers a means to avert their own human-centric biases and avoid the pitfall highlighted by Groom and Nass (2007): "While trying to make robots human, researchers have sometimes overlooked what makes robots special" (p. 494).

\section{Conclusion and Future Directions}

Here, we have argued to extend CASA to include scripts developed through interactions with media agents. We additionally proposed that the development of a media agentderived script is based on the social affordances of the media agent and temporal factors of relationships with media agents. The proposed extension broadens CASA's theoretical scope and reconciles CASA with findings that suggest people do not necessarily follow human-human social scripts when interacting with media agents.

Our extension accomplishes four goals. First, it reconciles the CASA perspective with trends in digital media use and accounts for some divergent research findings wherein human-media agent interactions are not consistent with human-human scripts (e.g., Edwards et al., 2019; Srinivasan \& Takayama, 2016; Takayama, 2015). Second, it increases the theoretical specificity of CASA by enabling it to account for another type of mindless interaction with media agents. Third, it mitigates some of CASA's anthropocentric bias. The fourth and crucial goal accomplished in extending CASA is promoting the examination of time as a variable in understanding the development and application of media-agent scripts.

A first step is to begin clarifying what type of scripts exist for media-agent interactions. Methodologically, identifying such scripts is likely to require more inductive approaches. As Guzman (2018) suggests, a diverse range of methods and methodologies is necessary to advance knowledge pertaining to human relationships with machines. Although CASA's experimental paradigm is useful for controlled comparisons, it is ill-suited for the exploratory research that may be required to elaborate human-media agent social scripts due to the emergent nature of scripts as well as their predicted nuance. To capture this nuance, inductive approaches may be adopted alongside traditional CASA experiments. For example, Edwards et al. (2019) analyzed open-ended responses in their experimental study. These qualitative responses suggested that interactions with a social robot elicited positive impressions through feelings of connectedness, while interactions with a human partner, following the same interaction script, felt impersonal and disconnected. Finally, given that time is a key factor in the development of scripts, we advocate for the use of longitudinal methods to test CASA's claims as well as our proposed extension.

As we develop more refined scripts through longer, more complex, and more variant interactions with media agents, these scripts may influence our interpersonal relationships. In the same way that human scripts are mindlessly applied to guide our interactions with media, over time, media scripts may be developed and mindlessly applied to our interactions with humans. A thorough and proper understanding of human communication processes and relationships may be informed by understanding how we interact with media agents. Through extension, inquiry within CASA's framework may suggest the reverse of its core prediction. Rather than treating computers like people, we may treat people like computers. 
In summary, CASA has been a productive framework for studying human-machine communication, human-computer interaction, human-robot interaction, and humanagent interaction. The extended CASA we proposed reconciles counterintuitive findings, acknowledges changes over the last three decades, encourages research from more diverse methodological approaches, and does not invalidate research findings within the CASA framework. HMC research guided by this extended CASA can inform a more robust understanding of humans, machines, communication, and the human-machine relationship.

\section{Acknowledgments}

No external funding supported this research. The authors would like to express their gratitude for the service of the reviewers and editorial team, whose suggestions markedly improved the paper. Additionally, the authors would like to thank Denise Solomon, Mary Beth Oliver, and Brandon Van Der Heide for their input and feedback at different stages of development.

\section{Author Biographies}

Andrew Gambino is a University Fellow and PhD Candidate in The Donald P. Bellisario College of Communications at Pennsylvania State University. He studies communication and relationships.

(D) https://orcid.org/0000-0001-8657-6788

Jesse Fox (PhD, Stanford University) is an Associate Professor in the School of Communication at Ohio State University. She researches all the things.

(1) https://orcid.org/0000-0002-5018-3250

Rabindra (Robby) A. Ratan (PhD, USC Annenberg) is an associate professor and AT\&T scholar in the Department of Media and Information at Michigan State University. Dr. Ratan conducts research on the effects of human-technology interaction, examining how media technologies (e.g., avatars, agents, automobiles) influence meaningful outcomes (e.g., persuasion, education, health/safety).

(D) https://orcid.org/0000-0001-7611-8046 


\section{References}

Ammari, T., Kaye, J., Tsai, J. Y., \& Bentley, F. (2019). Music, search, and IoT: How people (really) use voice assistants. ACM Transactions on Computer-Human Interaction, 26(3), article 17. https://doi.org/10.1145/3311956

Baxter, P., Ashurst, E., Read, R., Kennedy, J., \& Belpaeme, T. (2017). Robot education peers in a situated primary school study: Personalisation promotes child learning. PloS One, 12, e0178126. https://doi.org/10.1371/journal.pone.0178126

Baylor, A. L. (2011). The design of motivational agents and avatars. Educational Technology Research \& Development, 59, 291-300. https://doi.org/10.1007/s11423-011-9196-3

Beckhusen, J. (2016). Occupations in information technology. U.S. Census Bureau.

Bickmore, T., \& Cassell, J. (2001, March). Relational agents: A model and implementation of building user trust. In Proceedings of the SIGCHI conference on human factors in computing systems (pp. 396-403). ACM. https://doi.org/10.1145/365024.365304

Bickmore, T. W., \& Picard, R. W. (2005). Establishing and maintaining long-term humancomputer relationships. ACM Transactions on Computer-Human Interaction, 12(2), 293-327. https://doi.org/10.1145/1067860.1067867

Blascovich, J., Loomis, J., Beall, A. C., Swinth, K. R., Hoyt, C. L., \& Bailenson, J. N. (2002). Immersive virtual environment technology as a methodological tool for social psychology. Psychological Inquiry, 13, 103-124. https://doi.org/10.1207/S15327965PLI1302_01

Breazeal, C. (2003). Emotion and sociable humanoid robots. International Journal of Human-Computer Studies, 59, 119-155. https://doi.org/10.1016/S1071-5819(03)00018-1

Carolus, A., Schmidt, C., Schneider, F., Mayr, J., \& Muench, R. (2018). Are people polite to smartphones? Lecture Notes in Computer Science, 10902, 500-511. https://doi. org/10.1007/978-3-319-91244-8_39

Chiasson, S., \& Gutwin, C. (2005). Testing the media equation with children. In Proceedings of SIGCHI '05 Human Factors in Computing Systems (pp. 829-838). ACM. https://doi. org/10.1145/1054972.1055089

de Graaf, M. M., \& Allouch, S. B. (2013). Exploring influencing variables for the acceptance of social robots. Robotics and Autonomous Systems, 61, 1476-1486. https://doi. org/10.1016/j.robot.2013.07.007

de Graaf, M. M., Allouch, S. B., \& Van Dijk, J. A. (2019). Why would I use this in my home? A model of domestic social robot acceptance. Human-Computer Interaction, 34, 115173. https://doi.org/10.1080/07370024.2017.1312406

Edwards, A. (2018). Animals, humans, and machines: Interactive implications of ontological classification. In A. Guzman (Ed.), Human-machine communication: Rethinking communication, technology, and ourselves (pp. 29-50). Peter Lang.

Edwards, A., Edwards, C., \& Gambino, A. (2019). The social pragmatics of communication with social robots: Effects of robot message design logic in a regulative context. International Journal of Social Robotics. https://doi.org/10.1007/s12369-019-00538-7

Edwards, A., Edwards, C., Westerman, D., \& Spence, P. R. (2019). Initial expectations, interactions, and beyond with social robots. Computers in Human Behavior, 90, 308-314. https://doi.org/10.1016/j.chb.2018.08.042

Fogg, B. J., \& Nass, C. (1997). Silicon sycophants: The effects of computers that flatter. International Journal of Human-Computer Studies, 46, 551-561. https://doi.org/10.1006/ ijhc.1996.0104 
Fortunati, L. (2005). Is body-to-body communication still the prototype? The Information Society, 21, 53-61. https://doi.org/10.1080/01972240590895919

Fortunati, L. (2018). Robotization and the domestic sphere. New Media \& Society, 20, 26732690. https://doi.org/10.1177/1461444817729366

Fox, J., Ahn, S. J., Janssen, J. H., Yeykelis, L., Segovia, K. Y., \& Bailenson, J. N. (2015). Avatars versus agents: A meta-analysis quantifying the effect of agency on social influence. Human-Computer Interaction, 30, 401-432. https://doi.org/10.1080/07370024.2014.921 494

Fox, J., \& McEwan, B. (2017). Distinguishing technologies for social interaction: The Perceived Social Affordances of Communication Channels Scale. Communication Monographs, 84, 298-318. https://doi.org/10.1080/03637751.2017.1332418

Gibson, J. J. (1979). The ecological approach to visual perception. Houghlin Mifflin.

Gong, L. (2008). How social is social responses to computers? The function of the degree of anthropomorphism in computer representations. Computers in Human Behavior, 24, 1494-1509. https://doi.org/10.1016/j.chb.2007.05.007

Gong, L., \& Nass, C. (2007). When a talking-face computer agent is half-human and half-humanoid: Human identity and consistency preference. Human Communication Research, 33, 163-193. https://doi.org/10.1111/j.1468-2958.2007.00295.x

Gratch, J., Wang, N., Gerten, J., Fast, E., \& Duffy, R. (2007). Creating rapport with virtual agents. Lecture Notes in Computer Science, 4722, 125-138. https://doi.org/10.1007/9783-540-74997-4_12

Groom, V., \& Nass, C. (2007). Can robots be teammates? Benchmarks in human-robot teams. Interaction Studies, 8, 483-500. https://doi.org/10.1075/is.8.3.10gro

Guzman, A. L. (2018). Human-machine communication: Rethinking communication, technology, and ourselves. Peter Lang.

Hipple, S., \& Kosanovich, K. (2003). Computer and Internet use at work in 2001. Monthly Labor Review, 126, 26-35.

Ho, A., Hancock, J., \& Miner, A. S. (2018). Psychological, relational, and emotional effects of self-disclosure after conversations with a chatbot. Journal of Communication, 68, 712-733. https://doi.org/10.1093/joc/jqy026

Hoffmann, L., Krämer, N. C., Lam-Chi, A., \& Kopp, S. (2009, September). Media equation revisited: Do users show polite reactions towards an embodied agent? Lecture Notes in Computer Science, 5773, 159-165. https://doi.org/10.1007/978-3-642-04380-2_19

Honeycutt, J. M., \& Bryan, S. P. (2011). Scripts and communication for relationships. Peter Lang.

Horstmann, A. C., \& Krämer, N. C. (2019). Great expectations? Relation of previous experiences with social robots in real life or in the media and expectancies based on qualitative and quantitative assessment. Frontiers in Psychology, 10, article 939. https://doi. org/10.3389/fpsyg.2019.00939

Johnson, D., \& Gardner, J. (2007). The media equation and team formation: Further evidence for experience as a moderator. International Journal of Human-Computer Studies, 65, 111-124. https://doi.org/10.1016/j.ijhcs.2006.08.007

Kenny, P., Parsons, T., Gratch, J., \& Rizzo, A. (2008, July). Virtual humans for assisted health care. In Proceedings of the 1st International Conference on Pervasive Technologies Related to Assistive Environments (article 6). ACM. 
Kim, D. J., \& Lim, Y. K. (2019). Co-performing agent: Design for building user-agent partnership in learning and adaptive services. In Proceedings of SIGCHI 2019 Human Factors in Computing Systems. https://doi.org/10.1145/3290605.3300714

Krämer, N. C., Eimler, S., von der Pütten, A., \& Payr, S. (2011). Theory of companions: What can theoretical models contribute to applications and understanding of human-robot interaction? Applied Artificial Intelligence, 25, 474-502. https://doi.org/10.1080/088395 14.2011.587153

Krämer, N. C., von der Pütten, A., \& Eimler, S. (2012). Human-agent and human-robot interaction theory: Similarities to and differences from human-human interaction. In M. Zacarias \& J. V. Oliveira (Eds.), Human-computer interaction: The agency perspective (pp. 215-240). Springer.

Lee, E. J. (2009). I like you, but I won't listen to you: Effects of rationality on affective and behavioral responses to computers that flatter. International Journal of HumanComputer Studies, 67, 628-638. https://doi.org/10.1016/j.ijhcs.2009.03.003

Lee, E. J. (2010a). The more humanlike, the better? How speech type and users' cognitive style affect social responses to computers. Computers in Human Behavior, 26, 665-672. https://doi.org/10.1016/j.chb.2010.01.003

Lee, E. J. (2010b). What triggers social responses to flattering computers? Experimental tests of anthropomorphism and mindlessness explanations. Communication Research, 37, 191-214. https://doi.org/10.1111/j.1460-2466.2008.00386.x

Lee, K. M., Jung, Y., Kim, J., \& Kim, S. R. (2006). Are physically embodied social agents better than disembodied social agents? The effects of physical embodiment, tactile interaction, and people's loneliness in human-robot interaction. International Journal of Human-Computer Studies, 64, 962-973. https://doi.org/10.1016/j.ijhcs.2006.05.002

Lee, M. K., Forlizzi, J., Kiesler, S., Rybski, P., Antanitis, J., \& Savetsila, S. (2012, March). Personalization in HRI: A longitudinal field experiment. In Proceedings of HRI 2012 ACM/ IEEE International Conference on Human-Robot Interaction (pp. 319-326). IEEE.

Lee, S., Ratan, R., \& Park, T. (2019). The voice makes the car: Enhancing autonomous vehicle perceptions and adoption intention through voice agent gender and style. Multimodal Technologies and Interaction, 3, article 20. https://doi.org/10.3390/mti30100200

Lee, S. A., \& Liang, Y. (2016). The role of reciprocity in verbally persuasive robots. Cyberpsychology, Behavior, and Social Networking, 19, 524-527. https://doi.org/10.1089/ cyber.2016.0124

Lee, S. A., \& Liang, Y. (2019). Robotic foot-in-the-door: Using sequential-request persuasive strategies in human-robot interaction. Computers in Human Behavior, 90, 351356. https://doi.org/10.1016/j.chb.2018.08.026

Leite, I., Martinho, C., \& Paiva, A. (2013). Social robots for long-term interaction: A survey. International Journal of Social Robotics, 5, 291-308. https://doi.org/10.1007/s12369-0130178-y

Lewis, S. C., Guzman, A. L., \& Schmidt, T. R. (2019). Automation, journalism, and humanmachine communication: Rethinking roles and relationships of humans and machines in news. Digital Journalism, 7, 409-427. https://doi.org/10.1080/21670811.2019.1577147

McLean, G., \& Osei-Frimpong, K. (2019). Hey Alexa ... examine the variables influencing the use of artificial intelligent in-home voice assistants. Computers in Human Behavior, 99, 28-37. https://doi.org/10.1016/j.chb.2019.05.009 
Nass, C., \& Brave, S. (2005). Wired for speech: How voice activates and advances the human-computer relationship. MIT Press.

Nass, C., Fogg, B. J., \& Moon, Y. (1996). Can computers be teammates? International Journal of Human-Computer Studies, 45, 669-678. https://doi.org/10.1006/ijhc.1996.0073

Nass, C., Jonsson, I. M., Harris, H., Reeves, B., Endo, J., Brave, S., \& Takayama, L. (2005, April). Improving automotive safety by pairing driver emotion and car voice emotion. In CHI'05 Extended Abstracts on Human Factors in Computing Systems (pp. 1973-1976). ACM.

Nass, C., \& Moon, Y. (2000). Machines and mindlessness: Social responses to computers. Journal of Social Issues, 56, 81-103. https://doi.org/10.1111/0022-4537.00153

Nass, C., Moon, Y., \& Green, N. (1997). Are computers gender-neutral? Gender stereotypic responses to computers. Journal of Applied Social Psychology, 27, 864-876.

Nass, C., \& Steuer, J. (1993). Voices, boxes, and sources of messages: Computers and social actors. Human Communication Research, 19, 504-527. https://doi. org/10.1111/j.1468-2958.1993.tb00311.x

Nass, C., Steuer, J., \& Tauber, E. R. (1994). Computers are social actors. In Proceedings of SIGCHI '94 Human Factors in Computing Systems (pp. 72-78). ACM. https://doi. org/10.1145/259963.260288

Nowak, K. L., \& Fox, J. (2018). Avatars and computer-mediated communication: A review of the definitions, uses, and effects of digital representations. Review of Communication Research, 6, 30-53. https://doi.org/10.12840/issn.2255-4165.2018.06.01.015

Pew Research Center. (2019). Mobile technology and home broadband 2019. Pew.

Pfeifer, L. M., \& Bickmore, T. (2011, May). Is the media equation a flash in the pan? The durability and longevity of social responses to computers. In Proceedings of the SIGCHI Conference on Human Factors in Computing Systems (pp. 777-780). ACM.

Reeves, B., \& Nass, C. (1996). The media equation: How people treat computers, television, and new media like real people and places. Cambridge.

Rosenthal-Von Der Pütten, A. M., \& Krämer, N. C. (2014). How design characteristics of robots determine evaluation and uncanny valley related responses. Computers in Human Behavior, 36, 422-439. https://doi.org/10.1016/j.chb.2014.03.066

Ryan, C. L., \& Bauman, K. (2016). Educational attainment in the United States: 2016. U.S. Census Bureau.

Ryan, C. L., \& Lewis, J. M. (2017). Computer and internet use in the United States: 2016. U.S. Census Bureau.

Schank, R. C., \& Abelson, R. P. (1977). Scripts, plans, goals, and understanding: An inquiry into human knowledge structures. Erlbaum.

Serholt, S., \& Barendregt, W. (2016, October). Robots tutoring children: Longitudinal evaluation of social engagement in child-robot interaction. In Proceedings of the 9th Nordic Conference on Human-Computer Interaction (article 64). ACM. https://doi. org/10.1145/2971485.2971536

Shneiderman, B., Plaisant, C., Cohen, M. S., Jacobs, S. A., \& Elmqvist, N. (2017). Designing the user interface: Strategies for effective human-computer interaction (6th ed.). Pearson.

Spence, P. R. (2019). Searching for questions, original thoughts, or advancing theory: Human machine communication. Computers in Human Behavior, 90, 285-287. https:// doi.org/10.1016/j.chb.2018.09.014 
Spence, P. R., Westerman, D., Edwards, C., \& Edwards, A. (2014). Welcoming our robot overlords: Initial expectations about interaction with a robot. Communication Research Reports, 31, 272-280. https://doi.org/10.1080/08824096.2014.924337

Srinivasan, V., \& Takayama, L. (2016). Help me please: Robot politeness strategies for soliciting help from humans. In Proceedings of the SIGCHI '16 Human Factors in Computing Systems (pp. 4945-4955). ACM. https://doi.org/10.1145/2858036.2858217

Sundar, S. S., \& Nass, C. (2000). Source orientation in human-computer interaction: Programmer, networker, or independent social actor? Communication Research, 27, 683703. https://doi.org/10.1177/009365000027006001

Takayama, L. (2015). Telepresence and apparent agency in human-robot interaction. In S. S. Sundar (Ed.), The handbook of the psychology of communication technology (pp. 160-175). Wiley \& Sons.

Von der Pütten, A. M., Krämer, N. C., Gratch, J., \& Kang, S. H. (2010). It doesn’t matter what you are! Explaining social effects of agents and avatars. Computers in Human Behavior, 26, 1641-1650. https://doi.org/10.1016/j.chb.2010.06.012

Waytz, A., Cacioppo, J., \& Epley, N. (2010). Who sees human? The stability and importance of individual differences in anthropomorphism. Perspectives on Psychological Science, 5, 219-232. https://doi.org/10.1177/1745691610369336 

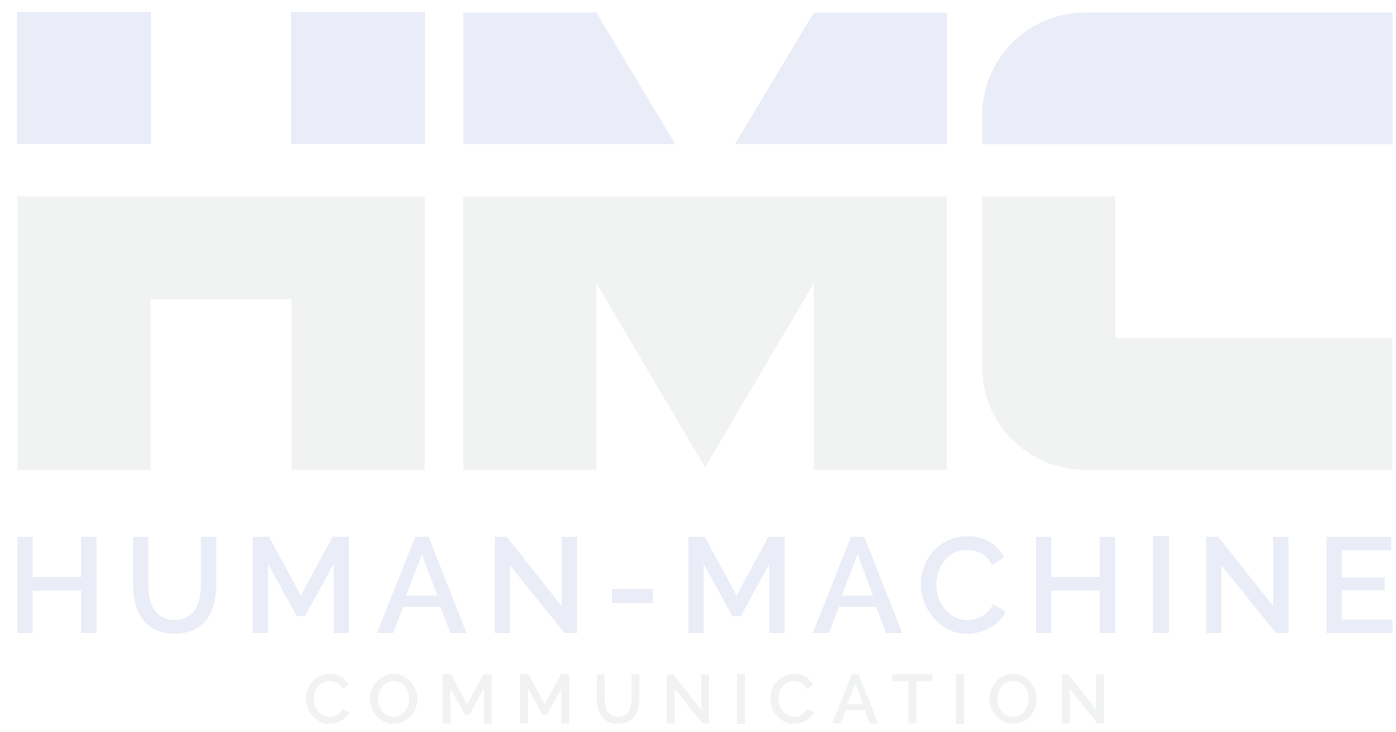